\title{
ASSOCIAÇÃO DE ALELOS DO COMPLEXO PRINCIPAL DE HISTOCOMPATIBILIDADE DE CLASSE II COM A DOENÇA DE GRAVES, EM PACIENTES BRASILEIROS
}

\author{
ASSOCIATION OF MAJOR HISTOCOMPATIBILITY COMPLEX CLASS II ALLELES \\ WITH GRAVES' DISEASE IN BRAZILIAN PATIENTS
}

Léa MZ Maciel ${ }^{1}$; Sandra S Rodrigues²; Ralph S Dibbern 3 ; Paula AA Navarro³ \& Eduardo A Donadi ${ }^{4}$

\begin{abstract}
${ }^{1}$ Docente da Divisão de Endocrinologia; ${ }^{2}$ Técnica do Laboratório de Biologia Molecular; ${ }^{3}$ Monitores da Divisão de Endocrinologia; ${ }^{4}$ Docente da Divisão de Imunologia Clínica. Departamento de Clínica Médica da Faculdade de Medicina de Ribeirão Preto-USP

CorRespondêncIA: Léa M Z Maciel - Departamento de Clínica Médica da Faculdade de Medicina de Ribeirão Preto - Av. Bandeirantes, 3900 - 14048-900 - Ribeirão Preto, SP - Fax: 16-6331144 - e-mail: Imzmacie@fmrp.usp.br
\end{abstract}

MACIEL LMZ; RODRIGUES SS; DIBBERN RS; NAVARRO PAA \& DONADI EA. Associação de alelos do complexo principal de histocompatibilidade de classe II com a Doença de Graves, em pacientes brasileiros. Medicina, Ribeirão Preto, 33: 42-46, jan./mar. 2000.

RESUMO: A Doença de Graves (DG) é a causa mais freqüente de hipertireoidismo. Sua origem é multifatorial, complexa, na qual a susceptibilidade genética interage com o meio ambiente e fatores endógenos para causar a doença. Os alelos do Complexo Principal de Histocompatibilidade $(\mathrm{CPH})$ de classe II têm sido associados com a DG, em populações de diferentes etnias e muitas evidências apontam para a associação do antígeno HLA-DR3 em caucasianos. O envolvimento dos alelos do $\mathrm{CPH}$ na nossa população, altamente miscigenada, foi estudado em pacientes com a DG e em indivíduos-controle da mesma área geográfica, utilizando-se DNA genômico amplificado e hibridado com iniciadores de seqüência específica (SSP). O alelo HLADRB $1{ }^{*} 0301$ esteve presente com maior freqüência $(44,7 \%)$ em pacientes com DG, relativamente à população-controle $(22,3 \%, p c=0,0068)$, conferindo Risco Relativo $(R R)$ de 2,8 e uma Fração Etiológica de 28,7, enquanto o alelo HLA-DQB1*0602 esteve, significantemente, diminuído nesses pacientes $(8 \%)$ em relação aos pacientes-controle $(31,9 \%, p c=0,00062)$, conferindo $R R$ de 1,8 e uma Fração Preventiva de 26,7. Apesar de a população brasileira ser altamente miscigenada, a confirmação do alelo HLA-DRB1*0301, conferindo susceptibilidade à doença, aponta esse alelo com um marcador importante na predisposição à doença. Em contraste, a proteção conferida pelo alelo $\mathrm{HLA}-\mathrm{DQB} 1{ }^{*} 0602$ parece ser peculiar aos pacientes brasileiros.

UNITERMOS: Doença de Graves. Complexo Principal de Histocompatibilidade. Associação. Alelos.

\section{INTRODUÇÃO}

Em áreas suficientes em iodo, a Doença de Graves (DG) é a causa mais comum de hipertireoidismo. A DG é uma doença auto-imune, órgão-específica, que incide, principalmente, em mulheres e apresenta importante agregação familiar. A síndrome é caracteri- zada por bócio difuso hiperplásico, podendo ser acompanhada por manifestações extratireóideas, como a oftalmopatia e o mixedema pré-tibial, que ocorrem, respectivamente, em 50 e $5 \%$ dos pacientes. Sua incidência é de, aproximadamente, 15-50:100.000 pessoas por ano e a relação mulher/homem, $7: 1^{(1,2,3)}$. Apesar de a doença incidir em todas as faixas etárias, ela 
é mais comum em adultos jovens entre a $3^{\mathrm{a}}$ e $4^{\mathrm{a}}$ décadas. Histologicamente, caracteriza-se pela infiltração linfocítica da glândula e, imunologicamente, pela evidência de ativação do sistema imune com níveis elevados de linfócitos $\mathrm{T}$ ativados na circulação e autoanticorpos específicos contra a tireóide. Pacientes com DG têm anticorpos contra diversos antígenos tireóideos. Os mais importantes são aqueles dirigidos contra o receptor de TSH (TSH-R), pois estão implicados na etiopatogênese da doença. A ativação desses receptores pelos anticorpos causa a síntese (via AMP-c) e a liberação dos hormônios e o hipertireoidismo clínico, além de promover o crescimento da glândula $^{(4,5)}$. Além desses, a maioria dos pacientes com DG têm anticorpos contra outros auto-antígenos, particularmente, a tireoglobulina e a peroxidase, esses com pequeno papel sobre a etiologia da doença, servindo apenas como marcadores de auto-imunidade e susceptibilidade. Recentemente, auto-anticorpos contra a proteína transportadora de iodo na membrana da célula tireóidea têm sido demonstrados em pacientes com DG e Tireoidite de Hashimoto, mas seu papel fisiopatológico não é conhecido ${ }^{(6)}$.

As evidências de um sistema imune alterado, encontradas nos pacientes com DG, vão além da presença de auto-anticorpos circulantes, e incluem a detecção de citocinas circulantes como as interleucinas $1,4,8,10$ e interferon $\gamma$ em níveis mais elevados do que o normal. A fonte destas citocinas circulantes é provavelmente a tireóide. Além disso, hiperplasia tímica tem sido relatada em pacientes com DG não trata$\mathrm{da}^{(7)}$. Nenhum antígeno anormal foi detectado na glândula de pacientes afetados e o envolvimento de múltiplos órgãos (tireopatia, oftalmopatia e dermopatia) sugere tratar-se de um defeito generalizado da regulação do sistema imune.

A DG inclui-se em um grupo de doenças complexas, apresentando, em comum, o modo de herança não mendeliana, poligênica (implicando no envolvimento de vários genes) e multifatorial. Fatores genéticos, constitucionais e ambientais parecem estar envolvidos na patogênese da doença, porém a contribuição relativa de cada um desses fatores permanece por ser determinada ${ }^{(5)}$.

\section{Fatores genéticos}

A importância dos fatores genéticos é ilustrada pela incidência de vários casos da doença em uma mesma família ${ }^{(8)}$, bem como pela concordância de sua ocorrência em $50 \%$ em gêmeos univitelinos e em $30 \%$ em bivitelinos.

\section{Fatores ambientais}

Dentre os fatores ambientais, merecem destaque a influência da concentração de iodo na die$\operatorname{ta}^{(9)}$, a participação de vírus e bactérias ${ }^{(10)}$, do estresse $^{(11,12)}$ e do fumo ${ }^{(13)}$.

\section{Iodo}

Ratos geneticamente susceptíveis desenvolvem tireoidite auto-imune, quando o iodo é suplementado na dieta. Dois mecanismos podem estar envolvidos: a maior iodinação da tireoglobulina, tornando-a mais antigênica, e a geração de radicais livres de oxigênio dentro da glândula, levando à toxicidade celular. Porém, estudos em humanos, realizados em áreas suficientes em iodo, não demonstraram relação entre a ingestão de iodo e a auto-imunidade ${ }^{(14)}$, havendo apenas a demonstração de aumento da incidência de Tireoidite de Hashimoto, desencadeada pela suplementação de iodo em população deficiente desse elemento $^{(15)}$

\section{Infecção}

Um processo infeccioso pode, potencialmente, iniciar um fenômeno auto-imune, se o agente infectante possuir antígenos semelhantes ao do hospedeiro para induzir uma reação cruzada. $\mathrm{O}$ agente mais estudado é a Yersinia enterocolitica, pois a imunização de animais com a proteína de superfície da bactéria induz a formação de anticorpos que apresentam reação cruzada com o domínio extracelular do TSH-R, além de existirem evidências de infeções subclínicas e persistentes com a Yersinia em alguns pacientes com DG (16). Com relação aos vírus, há relatos de que alguns deles podem infectar e transformar linhagens de células tireóideas de ratos. Além disso, existe evidência da participação dos vírus na patogenia da doença pelos relatos de detecção no soro de alguns pacientes com DG, de anticorpos contra o vírus Coxsachie B.

\section{Estresse}

Estudos controlados têm demonstrado que pacientes com DG, freqüentemente, referem fatores estressantes poucos meses antes do desencadeamento da doença. Os mecanismos patofisiológicos são obscuros, mas podem estar relacionados com alteração do eixo hipotálamo-hipófise-adrenal, durante ou após esses eventos, levando a uma supressão do sistema imune. Os efeitos potenciais do estresse na etiologia da DG permanecem desconhecidos. 


\section{Fumo}

Apesar de Prummer \& Wiersinga ${ }^{(13)}$ terem demonstrado que o fumo está associado com DG e, especialmente, com o aumento do risco para o desenvolvimento da oftalmopatia mais grave, não existem dados conclusivos quanto a essa possibilidade.

A partir da definição das bases imunológicas da doença ${ }^{(17)}$, surgiu o interesse em se caracterizar os genes reguladores da resposta imune e aqueles que expressam o antígeno-alvo, ou seja, o receptor do TSH. Esses estudos, inicialmente, focalizaram os genes do sistema HLA, cuja associação com a DG tem sido conhecida desde a década de $1970{ }^{(18)}$. Os pacientes com DG exibem expressão aberrante de antígenos do Complexo Principal de Histocompatibilidade (CPH) de classe II. Existem evidências crescentes, apontando a associação do antígeno HLA DR-3 com a DG, pelo menos em caucasianos, porém com um risco relativo baixo, variando de 3-5. O antígeno HLA-DR-3 está em forte desequilíbrio de ligação com aqueles codificados pelos alelos HLA-DQB1*0201 e DQA1*0501, também, freqüentemente, associados com a DG. A freqüência dos antígenos HLA entre os pacientes portadores da DG, no Brasil, foi relatada recentemente ${ }^{(19)}$, utilizando-se o método sorológico. Nesse trabalho, observou-se um aumento significante da especificidade HLA-DR3. As tipificações dos marcadores imunogenéticos, utilizando o DNA genômico ${ }^{(20)}$, tem demonstrado que o número de alelos HLA de classe II é maior que aquele detectado por métodos sorológicos. Assim, utilizando-se DNA genômico amplificado e hibridado com iniciadores de sequiência específica (sequence-specific primers-SSP) observamos que o gene HLA-DRB1*0301 esteve presente com maior freqüência $(44,7 \%)$ em pacientes com a DG em relação à população-controle $(22,3 \%$, pc $=0,0068)$, conferindo um Risco Relativo (RR) de 2,8 e uma Fração Etiológica de 28,7, enquanto que o alelo HLADQB1*0602 esteve, significantemente, diminuído nesses pacientes ( $8 \%$ ) em relação aos pacientes-controle $(31,9 \%$, pc $=0,00062)$, conferindo um RR de 1,8 e uma Fração Preventiva de 26,7, (Maciel et al, dados não publicados).

Em outras populações, a DG está associada aos antígenos HLA-A2, -A10, HLA-B8, -B46, HLA-DR1, -DR3,-DR9,-DQ2, e com os alelos DQB1*0303 e HLA-DPB $1 * 0501^{(21,22)}$. A Tabela I sumaria as associações entre os antígenos e os alelos de histocompatibilidade em diversas populações.
A confirmação do alelo HLA-DRB1*0301, conferindo susceptibilidade à doença, em nossa população, altamente miscigenada, aponta esse alelo como um marcador importante na susceptibilidade à doença. Em contraste, a proteção conferida pelo alelo HLADQB1*0602 parece ser peculiar aos pacientes brasileiros.

\section{Associação com marcadores não-HLA}

Entre os genes não-HLA, envolvidos na susceptibilidade à doença, estão os genes do antagonista do receptor da interleucina- $1^{(23)}$ e os genes $\alpha$ e $\beta$ do receptor da célula $T^{(24)}$, que têm sido objeto de particular interesse, devido ao papel central de seus produtos na resposta imune normal e também na resposta aberrante. Mais recentemente, tem sido dada grande atenção ao gene CTLA-4 (Citotoxic T lymphocyte antigen-4) $)^{(25)}$, proteína envolvida na apresentação do antígeno, como marcador do DG. A importância da participação desses genes na etiopatogenia da doença não está estabelecida.

$\mathrm{O}$ fato de a DG se desenvolver como resultado da presença de anticorpos estimuladores do TSH-R torna o gene para essa proteína um candidato óbvio para o desenvolvimento da doença. Um polimorfismo desse gene tem sido descrito no códon 52 do domínio extracelular do gene da molécula do receptor, causando a mudança do aminoácido prolina para treonina, podendo resultar em mudança da função do receptor e/ou em alteração na antigenicidade da proteína ${ }^{(26)}$. Apesar disso, os dois estudos relatados na literatura não demonstraram associação entre o gene do TSH$\mathrm{R}$ e o fenótipo da $\mathrm{DG}^{(27,28)}$.

Em trabalho recente, com o estudo de avaliação diferencial em larga escala de todo o genoma, foi relatado que a região no cromossoma 20q11.2 apresenta forte associação com DG, sugerindo serem os genes dessa região marcadores que conferem maior susceptibilidade à doença ${ }^{(29)}$.

\section{CONCLUSÃO}

DG é uma multifatorial, complexa, na qual a susceptibilidade genética interage com o meio ambiente e fatores endógenos para causar a doença. É provável que a DG, como outras doenças auto-imunes, órgão-específicas, seja poligênica, não havendo um único gene responsável o suficiente para que a doença se desenvolva. 
Tabela I - Associação de marcadores genéticos do CPH com a Doença de Graves

\begin{tabular}{|c|c|c|c|c|c|}
\hline \multicolumn{2}{|l|}{ Marcadores sorológicos } & \multicolumn{2}{|l|}{ HLA-DR } & \multicolumn{2}{|l|}{ HLA-B } \\
\hline \multicolumn{6}{|l|}{ SUSCEPTIBILIDADE } \\
\hline Brancos caucasianos & & \multicolumn{2}{|l|}{-DR3 } & \multicolumn{2}{|l|}{ B8 } \\
\hline Negros (África do Sul) & & \multicolumn{2}{|l|}{-DR3 } & & \\
\hline Japoneses & & \multicolumn{2}{|l|}{-DR5 } & \multicolumn{2}{|l|}{ B46 } \\
\hline Chineses & & \multicolumn{2}{|l|}{-DR9 } & \multicolumn{2}{|l|}{ B46, B5 } \\
\hline Coreanos & & \multicolumn{2}{|l|}{-DR5, DR8 } & \multirow{2}{*}{\multicolumn{2}{|c|}{ B13 }} \\
\hline $\begin{array}{l}\text { Brasileiros } \\
\text { (população miscigenada) }\end{array}$ & & $-\mathrm{DR} 3$ & & & \\
\hline \multicolumn{6}{|l|}{ RESISTÊNCIA } \\
\hline Brancos caucasianos & & \multicolumn{3}{|l|}{-DR5 } & \\
\hline \multirow{2}{*}{$\begin{array}{l}\text { Marcadores moleculares } \\
\text { e/ou sorológicos }\end{array}$} & \multicolumn{4}{|c|}{ Classe II } & \multirow{2}{*}{$\begin{array}{l}\text { Classe I } \\
\text { HLA-B }\end{array}$} \\
\hline & HLA-DPB1 & HLA-DQB1 & HLA-DQA1 & HLA-DRB1 & \\
\hline \multicolumn{6}{|l|}{ SUSCEPTIBILIDADE } \\
\hline Brancos caucasianos & *0402 & *0301 & *0501 & $\begin{array}{l}\text { DRB1 }{ }^{*} 0301, \\
\text { DRB3 }{ }^{*} 0101, \\
\text {-DR11 }\end{array}$ & $-B 8$ \\
\hline Chineses & & *0303 & & -DR9 & $-B 46$ \\
\hline Japoneses & & ${ }^{*} 0501$ & & & \\
\hline $\begin{array}{l}\text { Brasileiros } \\
\text { (população miscigenada) }\end{array}$ & & & & DRB $1{ }^{*} 0301$ & \\
\hline \multicolumn{6}{|l|}{ RESISTÊNCIA } \\
\hline Brancos caucasianos & & & *0201 & -DR7 & \\
\hline Japoneses & & *0501 & & & \\
\hline Chineses & & *0301 & *0401 & -DR12 & \\
\hline Brasileiros & & $* 0602$ & & & \\
\hline
\end{tabular}

MACIEL LMZ; RODRIGUES SS; DIBBERN RS; NAVARRO PAA \& DONADI EA. Association of major histocompatibility complex Class II alleles with Grave's Disease in brazilian patients. Medicina, Ribeirão Preto, 33: 42-46, jan./march 2000.

ABSTRACT: Graves'disease (GD) is the most frequent cause of hyperthyroidism. Although the etiology is not completely elucidated, there are several lines of evidence suggesting multifactorial mechanisms. Genetical, constitutional and environmental factors are involved in the pathogenesis. Major Histocompatibility Complex (MHC) Class II alleles have been associated with GD in several populations of distinct ethnic background and there is increasing evidence supporting an association between GD and HLA-DR3 in Caucasian populations. The MHC Classe II alleles were evaluated in our highly miscigenated population with GD and in control individuals of the same geographic area. MHC Class II genes were identified using PCR-amplified DNA hybridized with sequence-specific probes. HLA DRB1*0301 allele was significantly increased in patients $(44,7 \%)$ as compared with controls $(22,3 \%, \mathrm{pc}=0.0068)$, conferring a Relative Risk (RR) of 4.1 and an Etiologic Fraction of 28.7. HLA-DQB1*0602 allele was significantly decreased in patients $(\mathrm{pc}=0.00062)$, conferring an RR and a Preventive Fraction of 1.8 and 26.7, respectively. Although the Brazilian population is highly miscegenated, the results corroborated the participation of the allele HLA-DRB $1^{*} 0301$ as a susceptibility marker to GD. In contrast, the protection conferred by the HLA-DQB1 ${ }^{*} 0602$ allele seems to be peculiar to Brazilian patients.

UNITERMS: Grave's Disease. Major Histocompatibility Complex. Association. Alleles. 


\section{REFERÊNCIAS BIBLIOGRÁFICAS}

1 - BERGLUND J; CHRISTENSEN S \& HALLENGREN B. Total and age-specific incidence of Graves' thyrotoxicosis, toxic nodular goiter and solitary toxic adenoma in Malmo,1970-1974. J Intern Med 227: 137-141, 1990.

2 - FURSZYFER J; KURLAND L; WOOLNER LB; ELVEBACK LR \& MCCONAHEY W M. Graves'disease in Olmsted County, Minnesota, 1935 through 1967. Mayo Clin Proc 4 : 586596, 1970.

3 - GALOFRE J; GARDIA-MAYOR R; FLUITERS E; FERNANDEZCALVET L; REGO A; PARAMO C \& ANDRADE MA. Incidence of different forms of thyroid dysfunction and its degrees in an iodine sufficient area. Thyroidology 6: 49-54, 1994.

4 - WEETMAN AP \& MCGREGOR AM. Autoimmune thyroid disease: further developments in our understanding. Endocr Rev 15: 788-830, 1994

5 - PHILIPPOU G \& MCGREGOR AM. The aetiology of Graves'disease: what is the genetic contribution? Clin Endocrinol 48: 393-395, 1998.

6 - SPITZWEG D \& HEUFELDER AE. Update on thyroid sodium iodide symporter: a novel thyroid antigen emerging on the horizon. Eur J Endocrinol 137: 22-23, 1997.

7 - MCIVER B \& MORRIS JC. The pahogenesis of Graves'disease. Endocrinol Metab Clin North Am 27: 73-89, 1998.

8 - HARVALD B \& HAUGE MA. A catamnestic investigation of Danish twins. Dan Med Bull 3: 150-158, 1956.

9 - KOUTRAS DA; KARAIKOS KS; PIPERINGOS GD; KITSOPANIDES J; BOUKIS MA; MAKRIYANNIS D; SOUVATZOGLOUA; SFONTOURIS J; EVANGELOPOULUS K \& MOULOPOULUS SD. Treatment of endemic goitre with iodine and thyroid hormones, alone or in combination. Endocrinol Exp 20: 57-65, 1986.

10 - TOMER Y \& DAVIES T. Infection, thyroid disease, and autoimmunity. Endocr Rev 14: 107-120, 1993.

11 - CHIOVATO L \& PINCHERA. A stressful life events and Graves'disease. Eur J Endocrinol 134:680-682, 1996.

12 - CHROUSOS GP. The hipothalamic-pituitary-adrenal axis and immune-mediated inflamation. N Engl J Med 332: 1351-1362, 1995.

13 - PRUMMEL MF \& WIERSINGA WM. Smoking and risk of Graves' disease. JAMA 269:479-482, 1993.

14 - GARDNER D; CENTOR RM \& UTIGER RD. Effects of low dose oral iodide supplementation on thyroid function in normal men. Clin Endocrinol 28: 283-288, 1988

15 - HARACH H; ESCALANTE D; ONATIVIA A; LEDERER OUTES J; SARAVIA DAY E \& WILLIAMS ED. Thyroid carcinoma and thyroidites in an endemic goiter region before and after iodine prophylaxis. Acta Endocrinol 108: 55-60, 1985.

16 - LUO G; FAN J; SEETHARAMAIAH GS; DESAI RK; DALLAS JS; WAGLE N; DOAN R; NIELSEN DW; KLIMPEL GR \& PRABHAKAR BS. Immunization of mice with Yersinia enterocolitica leads to induction of antithyrotropin receptor antibodies. J Immunol 151: 922-928, 1993.
17 - MCGREGOR AM. Autoimmunity in the thyroid: can the molecular revolution contribute to our understanding?. Q J Med 82: 1-13, 1992.

18 - BECK K; LUMHOLTZ B; NERUP J; THOMSEN M; PLATZ P; RYDER LP; SVERJGAARD A; SIERSBAEK-NIELSEN K; HANSEN JM \& LARSEN JH. HLA antigens in Graves'disease. Acta Endocrinol 86: 510-516, 1977.

19 - MACIEL LMZ; NAVARRO PAA; RODRIGUES SS; IAZIGI N \& DONADI EA. Antígenos de Histocompatibilidade de Classe II em pacientes brasileiros portadores de Doença de Graves, com ou sem oftalmopatia. Arq Bras Endocrinol \& Metabol 41: 76-81, 1997.

20 - SVEJGAARD A \& RYDER LP. HLA and disease association: detecting the strongest association. Tissue Antigens 43: 18-27, 1994.

21 - BRIX TH; KYVIK KO \& HEGEDÜS L. What is the evidence of genetic factors in the etiology of Graves' disease? A brief review. Thyroid 8: 627- 634, 1998.

22 - MCLACHLAN SM \& RAPOPORT B. Genetics factors in thyroid disease. In: BRAVERMAN LE \& UTIGER RD, eds. Werner and ingbar's the thyroid: A fundamental and clinical text, 7th ed, Lippincott-Raven, Philadelphia, p.483-496, 1996.

23 - BLAKEMORE AI; WATSON PF; WEETMAN AP \& DUFF GW.. Assotiation of Graves' disease with an allele of the interleukin 1 receptor antagonist gene. J Clin Endocrinol Metabol 80: 111-115, 1995.

24 - DAVIESTF; MARTIN A; CONCEPTIONES; GRAVES P; COHEN $F$ \& BEN-NUN A. Evidence of limited variability of antigen receptors on intrathyrodal T cells in autoimmune thyroid disease. N Engl J Med 325: 238-244, 1991.

25 - YANAGAWA T; HIDAKA Y; GUIMARÃES V; SOLIMAN M \& DEGROOT LJ. CTLA-4 gene polymorphism associated with Graves'disease in Caucasian population. J Clin Endocrinol Metabol 80: 41-45,1995.

26 - BAHN RS; DUTTON CM; HEUFELDER AE \& SARKAR G. A genomic point mutation in the extracelular domain of the thyrotropin receptor in patients with Graves'ophtalmopathy. $\mathbf{J}$ Clin Endocrinol Metabol 78: 256-260,1994.

27 - TOMER Y; BARBESINO G; KEDDACHE M; GREENBERG DA \& DAVIES TF. Mapping of a maior susceptibility locus for Graves' disease (GD-1) to chromosome 14q31. J Clin Endocrinol Metab 82: 1645-1648, 1997.

28 - KOTSA KD; WATSON PF \& WEETMAN AP. No association between a thyrotropin receptor gene polymorphism and Graves'disease in female population. Thyroid 7: 31-33,1997.

29 - TOMER Y; BARBESINO G; GREENBERG DA; CONCEPCION E \& DAVIS TF. Mapping the major susceptibility loci for familial Graves' and Hashimoto's diseases: evidence for genetic heterogeneity and gene interactions. J Clin Endocrinol Metab 84: 4656-4664,1999.

Recebido para publicação em 14/01/2000

Aprovado para publicação em 03/03/2000 